Recepción: 05/11/2018

Aceptación: 10/01/2019

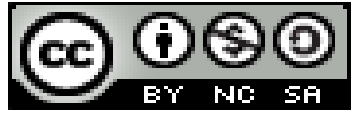

Ciencias de la computación

Publicación: 05/02/2019

Artículo de revisión

\title{
El analfabetismo tecnológico o digital
}

\section{Technological or digital illiteracy}

\section{Analfabetismo tecnológico ou digital}

\author{
Daniel Orlando Icaza-Álvarez ${ }^{\text {I }}$ \\ dicazaa@ucacue.edu.ec \\ Gerardo Eugenio Campoverde-Jiménez II \\ gcampoverdej@ucacue.edu.ec \\ Diego Efrain Verdugo-Ormaza ${ }^{\text {III }}$ \\ deverdugoo@ucacue.edu.ec \\ Pablo Danilo Arias-Reyes IV
pariasr@ucacue.edu.ec
}

Correspondencia:dicazaa@ucacue.edu.ec

\footnotetext{
${ }^{\text {I }}$ Magíster en Gestión de Telecomunicaciones, Especialista en Gerencia de Empresas de Telecomunicaciones, Ingeniero Eléctrico, Tecnólogo Industrial Especialidad Electromecánica, Docente de la Universidad Católica de Cuenca. Cuenca, Ecuador.

II Magíster en Seguridad, Salud y Ambiente, Ingeniero Eléctrico, Docente de la Universidad Católica de Cuenca. Cuenca, Ecuador.
III Magíster en Control y Automatización Industriales, Ingeniero Electrónico, Docente de la Universidad Católica de Cuenca. Cuenca, Ecuador.

IV Magíster en Sistemas Eléctricos de Potencia, Ingeniero Eléctrico, Docente de la Universidad Católica de Cuenca. Cuenca, Ecuador.
} 


\section{Resumen}

Para la elaboración del presente artículo, se asume que el desconocimiento de los avances tecnológicos o nuevas tecnologías se le conoce como analfabetismo digital, debido a que los individuos no tienen como interactuar con este tipo de herramientas y obtener mayores ventajas de las mismas, lo cual se traduce en el no manejo de un ordenador, del software, internet, y demás herramientas informáticas, factor que influye en el ámbito profesional, personal y social de cada persona. Al plantearse inicialmente un estudio sobre la influencia de los servicios de telecomunicaciones en las zonas rurales del cantón Cuenca-Ecuador, los autores determinaron la necesidad de establecer un piso teórico sobre el tema, y por ello el objetivo del presente, es exponer la revisión teórica hecha al respecto. La metodología utilizada fue el análisis del discurso. Se concluyó que si se sigue asociando alfabetización digital con planillas de cálculo, presentaciones y procesador de texto se tendrá una visión limitada del potencial de las nuevas tecnologías, los jóvenes basan las actividades tecnológicas a servicios colaborativos, redes sociales y a la comunicación por Internet, la cual no se limita solamente al correo electrónico, por lo que una vez más se recalca la importancia de que las personas sepan reconocer e identificar el potencial de las tecnologías propiciando así un verdadero alfabetismo tecnológico o digital.

Palabras Claves: Analfabetismo tecnológico; analfabetismo digital; telecomunicaciones en zona rural; nuevas tecnologías.

\section{Summary}

For the elaboration of this article, it is assumed that ignorance of technological advances or new technologies is known as digital illiteracy, because individuals do not have how to interact with this type of tools and obtain greater advantages from them, which it translates into the non-use of a computer, software, internet, and other computer tools, a factor that influences the professional, personal and social environment of each person. When initially considering a study on the influence of telecommunications services in the rural areas of the Cuenca-Ecuador basin, the authors determined the need to establish a theoretical floor on the subject, and therefore the objective of this is to present the theoretical review done about it The methodology used was discourse analysis. It was concluded that if digital literacy is still associated with spreadsheets,

\section{4}

Pol. Con. (Edición núm. 30) Vol. 4, No 2, febrero 2019, pp. 393-406, ISSN: 2550 - 682X 
presentations and word processing, there will be a limited vision of the potential of new technologies, young people base technological activities on collaborative services, social networks and Internet communication, the which is not limited only to electronic mail, so once again the importance is stressed that people know how to recognize and identify the potential of technologies thus promoting a true technological or digital literacy.

Keywords: Technological illiteracy; digital illiteracy; telecommunications in rural areas; new technologies

\section{Resumo}

Para a preparação deste artigo, presume-se que a falta de tecnologia ou novos desenvolvimentos de tecnologia é conhecida como a iliteracia digital, porque os indivíduos não têm como interagir com essas ferramentas e obter maiores vantagens dos mesmos, o que isso se traduz na não utilização de um computador, software, internet e outras ferramentas computacionais, fator que influencia o ambiente profissional, pessoal e social de cada pessoa. Ao considerar inicialmente um estudo sobre a influência dos serviços de telecomunicações nas áreas rurais do cantão Cuenca, Equador, os autores determinaram a necessidade de estabelecer um piso teórica sobre o assunto, e, portanto, o objetivo deste é para expor a revisão teórica feito sobre isso A metodologia utilizada foi a análise do discurso. Concluiu-se que, se a alfabetização digital ainda estiver associada a planilhas, apresentações e processamento de texto, haverá uma visão limitada do potencial das novas tecnologias, os jovens basearão atividades tecnológicas em serviços colaborativos, redes sociais e na comunicação pela Internet. que não se limita apenas ao correio eletrônico, então, mais uma vez, ressalta-se a importância de que as pessoas saibam reconhecer e identificar o potencial das tecnologias, promovendo assim uma verdadeira literacia tecnológica ou digital.

Palavras-chave: analfabetismo tecnológico; analfabetismo digital; telecomunicações nas zonas rurais; novas tecnologias

\section{Introducción}

En un estudio anterior, los autores abordaron la influencia de las telecomunicaciones en las comunidades rurales del Cantón Cuenca. La infraestructura y los sistemas de comunicación 
como, radio, televisión y teléfono, en las salas de reuniones, oficinas, escuelas y viviendas de campo que permiten a los ciudadanos estar al corriente de las novedades y participar con conocimiento de causa en las zonas rurales, estudiando la utilidad que presentan los Infocentros Comunitarios emplazados en determinados lugares como una posibilidad de acceso a la población rural del Cantón Cuenca, el cual con aproximación de 236.798 habitantes, en su mayoría trabajan en la agricultura y ganadería de subsistencia, el analfabetismo en gran proporción les impide adquirir los conocimientos necesarios para manejar la tecnología y les convierte en analfabetos digitales.

Es oportuno señalar que el desarrollo rural persigue dar respuesta a las necesidades básicas para hacer posible un futuro con más conocimientos en la tecnología, mejorar la formación de los estudiantes y el bienestar de los miles de personas que viven a nivel rural erradicando la pobreza extrema en las familias y evitando su migración a la urbe cuencana o inclusive fuera del país. Todos los grandes retos a los que se enfrenta hoy la humanidad para avanzar hacia el logro de un futuro sostenible ya sea conseguir la educación universal, reduciendo al máximo esa brecha digital es uno de los desafíos de la humanidad y particularmente del estado ecuatoriano mediante los distintos programas que procuran que la sociedad se inserte en el mundo del conocimiento, la igualdad entre los géneros, reducir la pérdida de biodiversidad y otros recursos medioambientales, etc.

Por otro lado, las teorías convencionales del desarrollo siguen buscando soluciones a los problemas de la pobreza mediante estrategias basadas en el aperturismo económico, la competitividad, la presencia del capital extranjero, la dolarización, la desregulación y flexibilización del mercado de trabajo, mediante intervenciones de carácter puntual, impulsadas fundamentalmente por organizaciones no gubernamentales y de cooperación internacional, que no han logrado los cambios suficientes para avanzar hacia la construcción de nuevas visiones y propuestas de desarrollo nacional y local.

El nivel de desconocimiento de las nuevas tecnologías impide que las personas puedan acceder a las posibilidades de interactuar con éstas, es decir, por una parte, navegar en la web, disfrutar de contenidos multimedia, socializar mediante las redes sociales, crear documentación, mediante la 
ejecución de políticas públicas, interpretar los planes, programas y proyectos que llevan adelante los GAD`s parroquiales, municipio de Cuenca o Prefectura del Azuay.

Es oportuno señalar que la proyección del Ministerio de Telecomunicaciones y de la Sociedad de la Información en el 2017 era contar con 1.400 Infocentros Comunitarios en el país, con una cobertura del $90 \%$ del total de cantones rurales. Además, se buscaba completar 24 MegaInfocentros, en las provincias de: Pichincha, Azuay, Cañar, Carchi, Cotopaxi, Chimborazo, El Oro, Esmeraldas, Guayas, Loja, Los Ríos, Manabí, Morona Santiago y Santo Domingo de los Tsáchilas, beneficiando a 1.696.414 ciudadanos, con una inversión social de 2.046.238.32 dólares; es decir, 85.259,26 dólares por cada MegaInfocentro. Si bien en varios sectores han sido de un gran beneficio, no es menos cierto que también en su gran mayoría han sido implementaciones de infraestructura física antes que cumplir su real objetivo el de incorporar a toda la zona rural a la sociedad del conocimiento.

Los nuevos servicios avanzados que pueden ofrecerse a través de las Nuevas Tecnologías son un elemento clave para conseguir la cohesión territorial, económica y social. Pero el aprovechamiento de las oportunidades que ofrecen las TIC no se producirá de forma automática, sino que debe ser impulsado por las instituciones públicas, por las empresas tecnológicas y los propios habitantes de las zonas rurales. Es decir, se debe ver el acceso a las nuevas tecnologías de la Información y comunicación como un derecho más de los ciudadanos.

La implantación de las TIC en un territorio está basada en tres pilares fundamentales: la infraestructura, la formación de la población en el uso de la tecnología y los servicios ofertados a través de ella. Estos tres pilares son complementarios y la mala implantación de cualquiera de las tres supone no poder aprovechar las posibilidades que ofrecen las Nuevas Tecnologías.

La mayoría de los niños hoy en día aprenden a usar las TIC y a conectarse a Internet a edades muy tempranas, y lo hacen en su mayoría solos o con la ayuda de amigos, con muy poca tutela paterna o escolar, esto es a menudo causado por la falta de conocimientos sobre las nuevas tecnologías de la mayoría de adultos, particularmente en lo que respecta a su alcance, ámbito y funciones más allá de los usos tradicionales, los niños y jóvenes usan las redes sociales y plataformas de mensajería instantánea para comunicarse con amigos conocidos y desconocidos, la mayoría de los adolescentes son parte al menos de una red social, donde casi siempre publican 
información personal tal y como sus nombres completos, dirección de su casa y escuela y número de teléfono.

Además, la mayoría de ellos comparten también en estos medios imágenes comprometedoras de ellos/as mismos/as, para atraer la atención y los comentarios de otros usuarios y pares, la popularidad se mide a menudo por el número de amigos que un individuo tiene en estas redes sociales, por lo que es una práctica común entre los adolescentes el aceptar entre sus amigos a desconocidos, en algunos casos incluso conociéndolos en la vida real posteriormente. Los cuidadores, como padres y maestros, generalmente están totalmente ausentes de las interacciones en línea de los adolescentes.

Es así que cada cosa que se exprese a través de las redes sociales suele tener un efecto positivo o negativo en los usuarios, dependiendo del contenido o la situación en que se dé, sin embargo se puede inferir, por previo conocimiento o experiencia propia, que la exposición abierta de los sentimientos de los jóvenes, aún más de los adolescentes, tiende a crear conflictos por el mismo hecho de que estas redes muchas de las veces facilitan un acceso ilimitado e incontrolable de personas, manipulación y chantajes emocionales, puede darse tal cantidad de imposturas, cambios de género y falseamiento de identidad que las relaciones verdaderas suelen ser difíciles de crear y mantener.

Los aportes recolectados en torno a los efectos o impactos que pueden tener las redes sociales o el uso del Internet en el desarrollo de las relaciones interpersonales de los jóvenes que los utilizan, es amplia y determinista, aunque resultaría injusto afirmar que el efecto globalizador de estas Tecnologías de la Información y Comunicación son totalmente dañinas, así por ejemplo, se puede destacar que gracias a ellas los usuarios pueden contactarse con sus familiares o amigos a nivel mundial de manera fácil, económica e incluso cómoda, pues lo pueden hacer desde la comodidad de su dormitorio y a cualquier hora del día.

Se requiere una capacidad de inmersión mental en el entorno y, además, el aprendizaje y desarrollo de diversas capacidades de acción en él, tanto en la relación alumno-profesor como en las relaciones de los alumnos y profesores entre sí. En consecuencia, las redes educativas 
telemáticas han de soportar procesos de interacción con imágenes, sonidos y textos, para lo cual es imprescindible la banda ancha.

Para poder conocer de qué manera se puede potenciar las tecnologías en favor de la educación y de su evolución, es menester conocer los aspectos positivos que se han podido encontrar hasta ahora de estas tecnologías que puedan ser aplicadas al área específica de la educación. La cantidad y tipo de factores positivos que se puedan encontrar son muy variados, resalta por su capacidad de adaptación, y, por consiguiente, de evolución social. Nos dice que un aspecto positivo de las tecnologías en la educación es que desarrolla nuevas destrezas y habilidades en sus usuarios, a las cuales, aquellos que desconocen dichas tecnologías, no se han adaptado y no son parte de ese desarrollo social, desde esta perspectiva, la tecnología se ofrece como una forma de desarrollarnos intelectualmente, sobre la cual se debe desarrollar nuevos procesos educativos este es el punto central en lo que se refiere a la educación, e incluso a la formación, porque la educación habrá de ser estrictamente interactiva en el espacio electrónico, si de verdad queremos denominarla educación.

Por todo lo anterior, y como se ha mencionado, a continuación, se presentan algunas consideraciones teóricas, con la finalidad de poder minimizar el analfabetismo digital, causa y motivo que no permite que las personas puedan aprovechar los nuevos recursos tecnológicos que existen.

\section{¿Qué es un analfabeto digital?}

Según Area (2000) en siglos anteriores (XIX y XX) se entendía alfabetizada a aquella persona que sabía leer y escribir, hoy en día, donde la comunicación se produce no solo a través del lenguaje escrito el concepto de alfabetización cambia completamente. En la actualidad el dominio solo de la lectoescritura parece insuficiente, ya que solo permite acceder a una parte de la información vehiculada en nuestra sociedad: aquella que esta accesible a través de los libros. Una persona analfabeta tecnológicamente queda al margen de la red comunicativa que ofertan las nuevas tecnologías. De lo anterior podemos desprender que los analfabetos digitales son todas aquellas personas que desarrollan sus actividades personales y profesionales sin vincularse con tecnologías o medios digitales, limitando sus procesos a recursos tradicionales y concretos, principalmente relacionados a la lectura y escritura, como el lápiz y al papel. 
Es importante mencionar, para efectos de dimensionar la influencia de este grupo, que según Díaz y Leitao (2005) al año 2005 en nuestro país más de un 95\% de la población era considerada analfabeta digital, este grupo, los divide en dos subgrupos, el primero, aquellos analfabetos por imposibilidad de acceso, ya sea por ubicación geográfica o de recursos; y el segundo, analfabetos con acceso a las tecnologías en distintos niveles, y que por opción o por no desarrollar competencias se han mantenido al margen de las tecnologías.

Algunas características generales son una fuerte resistencia a incorporar las tecnologías a sus distintos procesos, validando medios tradicionales como los únicos realmente efectivos para la realización de sus tareas. La habilidad por sí sola para manejar herramientas e instrumentos tecnológicos, teléfonos móviles, iPods, PC, etc. no da la potestad de ser un alfabeto Digital, es posible plantear que las competencias básicas, ya sea hacer una presentación o escribir un documento con el procesador de texto en el computador no son suficientes para superar el analfabetismo digital o tecnológico.

Según Pérez (2009), la brecha nativa/analfabeto genera el no aprovechamiento de las tecnologías que manejan nuestros estudiantes, las cuales pueden ser realmente efectivas para el cumplimento de los objetivos del curriculum educacional, el problema reiterado es que docentes y directivos no son capaces de tomar esta ventaja, incluso llegando a ver estas tecnologías como enemigas.

\section{La brecha digital}

La alfabetización digital capacita a las personas de la Sociedad del Conocimiento a un entorno de nuevas formas de comunicarse y de conseguir información para transfórmala en conocimiento, pero se producen desequilibrios en el acceso a las tecnologías digitales por parte de las personas más desfavorecidas produciéndose desigualdades sociales.

Según Ballestero (2002), a lo largo de la historia se han encontrado desigualdades y exclusiones sociales. La revolución industrial trajo el progreso con la incorporación de las máquinas a la actividad industrial y a la propia agricultura, pero también trajo más desigualdad. Los tiempos cambian y los trabajadores se adaptan a las nuevas máquinas para poder trabajar con ellas y también trabajar en las nuevas industrias que producen nuevas máquinas. La migración y 
la exclusión social en la historia de la humanidad no se acaban con la sociedad industrial, se acentúa en la transformación en sociedad de la información.

Para Gutiérrez (2003), la revolución digital, del inicio del tercer milenio, da lugar a lenguajes, formas de comunicar y entornos nuevos que requieren de nuevas destrezas por nuestra parte, sobre todo como usuarios que están en contacto con los medios y las formas de comunicarse que van apareciendo en nuestras vidas, obligados a aprender a leer y escribir multimedia.

Según la definición de la UNESCO (citada por Gutiérrez, 2003), es analfabeta funcional la persona que no puede emprender aquellas actividades en las cuales la alfabetización (saber leer y escribir) es necesaria para la actuación eficaz en su grupo y comunidad. Según esto, una persona se considera alfabetizada cuando en su vida cotidiana puede leer y escribir, comprendiéndola, una oración corta y sencilla. La alfabetización funcional se refiere a aquellas personas que pueden realizar todas las actividades necesarias para el funcionamiento eficaz de su grupo y comunidad, y que además les permite continuar usando la lectura, la escritura y el cálculo para su propio desarrollo y el de su comunidad.

Los aspectos claves de la alfabetización digital son el multimedia y la digitalización de la Sociedad de la Información donde se hace imprescindible leer y escribir multimedia. La información en los documentos multimedia se encuentra a través de textos, sonidos, gráficos e imágenes en movimiento. En la actualidad la forma que se produce la información y se accede a ella ha pasado de la forma impresa en papel a estar más relacionada con la digitalización, con bases de datos electrónicas y redes de intercambio y distribución de documentos multimedia local y globalmente. Estos cambios exigen nuevas destrezas técnica y de interpretación para crear y acceder al saber, exigiendo nuevos conocimientos simbólicos para las nuevas formas de comunicar. La integración de texto, sonido e imagen en los documentos multimedia, junto con la interactividad, hacen de este lenguaje algo específico que nos obliga a considerar ya la alfabetización digital o multimedia como una necesidad de hoy y algo imprescindible (Gutiérrez, 2003).

Según Serrano y Martínez, (2003). La Brecha Digital, en inglés Digital Divide, aparece cuando las desigualdades sociales surgen a medida que se desarrolla el uso de las Tecnologías digitales como el ordenador, Internet. El término opuesto al de brecha digital que se emplea con más 
frecuencia es el de inclusión digital. La brecha digital se define como la separación que existe entre las personas (comunidades, estados, países...) que utilizan las TIC como una parte rutinaria de su vida diaria y aquellas que no tienen acceso a las mismas y que, aunque las tengan no saben cómo utilizarlas.

La OECD (2001) indica que la Brecha Digital se refiere al desfase o división, entre individuos, hogares, áreas económicas y geográficas con diferentes niveles socioeconómicos con relación a sus oportunidades de acceso a las TIC, como al uso de Internet para una amplia variedad de actividades.

Sánchez Caballero, (2010) se refiere a la diferencia tecnológica entre comunidades que tienen acceso a las Tecnologías de la Información y aquellas que no. Por Tecnologías de Información entendemos el teléfono móvil, ordenadores, banda ancha, Internet y el software. Las diferencias pueden ser de tipo socioeconómico o sobre la capacidad para utilizar las Tecnologías de la Información de forma eficaz, debido a los distintos niveles de alfabetización y discapacidades.

Hay ciudadanos que voluntariamente rechazan su incorporación al uso de las tecnologías digitales, porque no están interesados o no lo encuentran necesario. Existe una brecha digital que es la diferencia entre los que no tienen y los que tienen. El uso de las TIC se va generalizando en la vida cotidiana y hay personas que van quedándose al margen, corriendo el riesgo de exclusión social, ya que de alguna manera se encuentran fuera de las oportunidades vitales que definen las conquistas de la ciudadanía social (Ballestero, 2002).

Ballestero (2002) indica que la Brecha Digital está relacionada con cuatro elementos: la disponibilidad de hardware, dispositivos que permitan acceder a Internet (información y comunicación); la posibilidad de conectarse desde cualquier lugar (hogar, trabajo, una oficina, etc.); el conocimiento de las herramientas para poder acceder y navegar en la red; y la capacidad para hacer que la información accesible se convierta en conocimiento. A estos cuatro factores habría que añadir el factor de la accesibilidad universal y el diseño para todos para que las personas con problemas de visión, oído, cognitivos y físicos no tengan ningún tipo de barreras y puedan acceder a la información y la comunicación a través de las tecnologías digitales.

\section{Conclusiones}


Como conclusiones del estudio se puede afirmar que dada la velocidad con la que avanzan las tecnologías es probable que la brecha digital siga aumentando, por lo que las posibilidades de alfabetizar digitalmente a las personas se hacen más complicadas cada día que pasa, por lo que el enfoque ha de ser diferente, no debe centrarse en las herramientas tecnológicas, por el contrario, debe centrarse en el proceso de construcción de aprendizaje, logrando que sean capaces de identificar y aceptar los cambios.

En relación a la utilización de los servicios de telecomunicaciones donde acceden los adultos mayores se puede concluir que, si bien ellos estarían aprovechando mejor estos recursos que brindan los servicios, pero que encuentran una barrera bastante grande cuando entran en contacto, ya que inclusive desde el manejo de sus dedos hasta poder encontrar una lógica en el manejo de los dispositivos electrónicos puede tardar algún tiempo y en muchos de los casos decepción por no lograr mayor destreza en su manejo.

Los jóvenes tienen más y distintas competencias tecnológicas, incomprendidas en muchos casos, pero que es posible estimular su uso para el logro de un verdadero aprendizaje, ya que lo importante en estos tiempos no es solo la información, si no la capacidad de procesar la misma, el cual alcanza su óptimo potencial con la utilización de las nuevas tecnologías de información y comunicación.

Como ya se comentó el desarrollo rural persigue dar respuesta a las necesidades básicas para hacer posible un futuro con más conocimientos en la tecnología, mejorar la formación de los estudiantes y el bienestar de los miles de personas que viven a nivel rural. Esto significa afrontar grandes retos para avanzar hacia el logro de un futuro sostenible reduciendo al máximo la brecha digital, procurando que la sociedad se inserte en el mundo del conocimiento.

Además, se puede destacar que existe sobre el ser humano un gran conjunto de factores que influyen sobre su comportamiento, en el caso que no ocupa, nos hemos enfocado en los factores familiares, sociales, emocionales y formativos. Dentro de este grupo, resalta uno que está acaparando cada vez mayor atención, se trata de las redes sociales. Estos espacios de vinculación social, son para el adolescente y joven, una plataforma donde pueden converger con sus amistades y compartir momentos de esparcimiento, sin embargo, esto puede ser un factor negativo cuando no existen los cuidados pertinentes. 
Es importante que los adolescentes aprovechen el internet para informase y descargar programas que los ayuden a la comunicación, y aprovechar los tiempos libres para no encerrarse solo en la tecnología que puede provocar que se encierren en un mundo en que se pierde la importancia de compartir con los demás.

Resultaría interesante que los programas de formación y orientación estudiantil que se da en los colegios, se direccionen hacia la planificación de actividades que impulsen el contacto de los adolescentes con el medio que los rodea, ya sea mediante ayuda social a la comunidad, convivencias con sus compañeros, actos recreativos con sus familiares, etc.

Todo lo anterior exige una atención prioritaria al desarrollo con el acceso a los servicios básicos que ofrece la tecnología, ya que es el nivel de desconocimiento de las nuevas tecnologías lo que impide que las personas puedan acceder a las posibilidades de interactuar con éstas, es decir, por una parte navegar en la web, disfrutar de contenidos multimedia, socializar mediante las redes sociales, crear documentación, mediante la ejecución de políticas públicas, interpretar los planes, programas y proyectos que llevan adelante los GAD`s parroquiales, municipio de Cuenca o Prefectura del Azuay.

Ha quedado claro que, los servicios avanzados que pueden ofrecerse a través de las Nuevas Tecnologías son un elemento clave para conseguir la cohesión territorial, económica y social y que el aprovechamiento de las oportunidades que ofrecen las TIC no se producirá de forma automática sino que debe ser impulsado por las instituciones públicas, por las empresas tecnológicas y los propios habitantes de las zonas rurales, viendo a las nuevas tecnologías de la Información y comunicación como un derecho más de los ciudadanos.

Es muy importante recalcar que, la implantación de las TIC está basada en tres pilares fundamentales: la infraestructura, la formación de la población en el uso de la tecnología y los servicios ofertados a través de ella. Estos tres pilares son complementarios y la mala implantación de cualquiera de las tres supone no poder aprovechar las posibilidades que ofrecen las Nuevas Tecnologías.

Por ello, para poder conocer de qué manera se puede potenciar las tecnologías en favor de la educación y de su evolución, es necesario conocer los aspectos positivos de estas tecnologías. La 
cantidad y tipo de factores positivos que se puedan encontrar resalta por su capacidad de adaptación, y, por consiguiente, de evolución social.

Está claro que un aspecto positivo de las tecnologías en la educación es que desarrolla nuevas destrezas y habilidades en sus usuarios, a las cuales, aquellos que desconocen dichas tecnologías, no se han adaptado y no son parte de ese desarrollo social, desde esta perspectiva, la tecnología se ofrece como una forma de desarrollarnos intelectualmente, sobre la cual se debe desarrollar nuevos procesos educativos este es el punto central en lo que se refiere a la educación, e incluso a la formación, porque la educación habrá de ser estrictamente interactiva en el espacio electrónico, si de verdad queremos denominarla educación.

El uso del Internet en el desarrollo de las relaciones interpersonales de los jóvenes que los utilizan, es amplia y determinista, aunque resultaría injusto afirmar que el efecto globalizador de estas Tecnologías de la Información y Comunicación son totalmente dañinas, así por ejemplo, se puede destacar que gracias a ellas los usuarios pueden contactarse con sus familiares o amigos a nivel mundial de manera fácil, económica e incluso cómoda, pues lo pueden hacer desde la comodidad de su dormitorio y a cualquier hora del día. Algunos de los impactos de las redes electrónicas en las relaciones personales o comerciales.

Por último, si se sigue asociando alfabetización digital con planillas de cálculo, presentaciones y procesador de texto se tendrá una visión limitada del potencial de las nuevas tecnologías, los jóvenes basan en su gran mayoría las actividades tecnológicas a servicios colaborativos, redes sociales y a la comunicación por Internet, la cual no se limita solamente al correo electrónico, por lo que una vez más se recalca la importancia de que las personas sepan reconocer e identificar el potencial de las tecnologías propiciando así un verdadero alfabetismo tecnológico o digital.

\section{Referencias Bibliográficas}

Area, Manuel. (2001) Sociedad de la información y analfabetismo tecnológico. Revista Diálogos. N.p., n.d. Web.

Ballestero, F. (2002. La Brecha Digital: El Riesgo de Exclusión en la Sociedad de la Información. Madrid. Fundación Retevisión. 
Díaz, A y Leitao, P. (2005).Analfabetismo Digital. Concepción Publicación de la Universidad de Concepción.

Gutiérrez, M. (2003). Alfabetización Digital. Algo más que ratones y teclas. Barcelona. Editorial Gedisa.

OECD (2001) Understanding the Digital Divide. París. OECD (Organisation for Economic CoOperation and Development

Pérez, I, (2009) Tecnología Enemiga, El Miedo a lo desconocido. Recuperado de http://aulamagica.wordpress.com

Sánchez, M. (2010) Software libre y accesibilidad. Recuperado de http://www.nosolousabilidad.com/articulos/software_libre.htm

Serrano, a y Martínez, E (2003). La brecha digital: Mitos y realidades. México. Editorial UABC. 